\title{
Repubikla, un experimento participativo y laboratorio de datos para la movilidad activa
}

\author{
Celine Jacquin*
}

Recibido el 18 de marzo de 2016; aceptado el 20 de septiembre de 2016

\section{Résumé}

Dans un contexte où la gestion de l'information publique pâtit d'un manque général de ressources financières et de capacités techniques et institutionnelles, le manque de données et la rétention sont la règle. L'analyste du territoire souffre de cette absence et manuqe de robustesse de l'information, plus encore sur le thème de la mobilité urbaine. C'est dans ce contexte qu'a émergé le projet Repubikla, comme plateforme cartographique et groupe de travail indépendant, comme experimentation de crowdsourcing et de participation citoyenne.

Le projet cherche à permettre l'observation et la veille citoyenne pour promouvoir de meilleures conditions de mobilité active en ville, et d'un changement de paradigme sur le long terme, autour des méthodes de production de données sur la ville.

Le dévelopement de l'application web de cartographie volontaire et le travail de socialisation nécessaire pour la construction de la base de données, a vu émerger un groupe de travail dans lequel la diversité des profils et de l'expérience contribue positivement au développement du projet, son exposition et sa croissance vers de nouvelles problématiques.

Most-clés: crowdsourcing, cartographie participative, movilité active, activisme, politique publique.

\section{Resumo}

Em um contexto onde a gestão da informação pública sofre por falta de recursos e capacidades técnicas reduzidas, o atraso e a retenção de dados são a regra. O usuá-

* Geógrafa, colaboradora de OpenStreetMap, México, correo electrónico: celija@gmail.com. Los comentarios sobre este texto pueden realizarse en el blog del proyecto: <http://www. openstreetmap.mx/2016/08/crowdsourcing-movilidad-activa/>. 
rio analista de dados relacionados com o território e a população, se depara com a ausência de informação, em particular com o tema da mobilidade urbana. Nesta situação nasce o projeto Repubikla, como grupo de trabalho independente, experimento de crowdsourcing geográfico, participação cidadã, e plataforma de mapeamento. A visão geral do projeto aponta a observação cidadã a favor de melhores condições para a mobilidade ativa nas cidades, para a longo prazo gerar um processo de troca de paradigma ao redor da produção de dados sobre a cidade.

A experiência ao redor da aplicação de mapeamento voluntário, e o trabalho necessário de socialização para se lograr alimentar a base de dados, através de formação progressiva de um grupo de trabalho, em que a variedade de perfis e de experiências contribui positivamente para o desenvolvimento do projeto, a sua exposição, e a seu crescimento com novos temas, os quais antecedem ou se relacionam com o tema de origem: documentar as condições do ciclismo urbano.

Palavras chave: crowdsourcing, mapeamento, mobilidade ativa, ativismo, politica pública.

\section{Resumen}

En un contexto donde la gestión de la información pública adolece de recursos y capacidades técnicas reducidas, el rezago y la retención de datos son la regla. El usuario analista de datos relacionados con el territorio y la población, se enfrenta con la ausencia de información, en particular en el tema de la movilidad urbana. En esta situación nace el proyecto Repubikla, como grupo de trabajo independiente, experimento de crowdsourcing geográfico, participación ciudadana, y plataforma de mapeo. La visión general del proyecto apunta a la observación ciudadana a favor de mejores condiciones para la movilidad activa en las ciudades, para en el largo plazo, generar un proceso de cambio de paradigma alrededor de la producción de datos sobre la ciudad.

La experiencia alrededor de la aplicación de mapeo voluntario, y el trabajo necesario de socialización para lograr alimentar la base de datos, vio la formación progresiva de un grupo de trabajo, en el que la variedad de perfiles y de experiencias contribuye positivamente al desarrollo del proyecto, a su exposición y a su crecimiento hacia nuevos temas, los cuales anteceden o se relacionan con el tema de origen: documentar las condiciones del ciclismo urbano.

Palabras clave: crowdsourcing, mapeo, movilidad activa, activismo, política pública.

\section{Impulsar una reflexión metodológica de fondo en las instituciones}

El proyecto emerge dentro de un micro departamento de investigación aplicada, de una organización no gubernamental, que concentraba parte de sus acciones en el 
desarrollo y seguimiento de observatorios de movilidad sustentable. El equipo constituido por un desarrollador de sistemas computacionales y una geógrafa, experimentaba de manera continua el fuerte rezago de datos estandarizados, actuales y robustos, para estudiar la movilidad en general y en particular la movilidad no motorizada. La falta de información en este campo de estudio obliga al sector completo del urbanismo y de los estudios de movilidad al desarrollar proxys y métodos de estimación para lograr acercarse al tema; o simplemente imposibilita la observación, el análisis y la evaluación, limitando de manera crítica el impacto de la planeación urbana. En paralelo al desarrollo de metodologías que sólo logran ser paliativas, iniciamos una reflexión general sobre la eficiencia en la generación de datos públicos, y sobre la movilización de métodos basados en nuevas fuentes, como la aportación voluntaria de información, con un fuerte interés en métodos crowdsourced - pasivos o activos-, que a través de los teléfonos móviles representan un fuerte potencial para generar información sobre la movilidad urbana cotidiana.

Sin tener una solución ad-hoc, se buscaba iniciar un trabajo de gestión permanente con instituciones de gobierno a cargo de la producción de información geoespacial en México. Se buscaba impulsar la emergencia de foros de reflexión sobre nuevos métodos, y desarrollar la reflexión colectiva sobre los fundamentos de la producción de datos públicos. Apoyados por pocas personas la ONG, inició por un lado un acercamiento con el Instituto Nacional de Estadística y Geografía (INEGI) para tratar de influir en la actualización de la encuesta de movilidad del Valle de México, la cual no ha sido aplicada desde el 2007; y en segundo lugar, para impulsar una reflexión compartida acerca de mecanismos permanentes de investigación sobre modalidades voluntarias de creación de información para cualquier tipo de encuestas masivas.

En términos metodológicos y conceptuales, el crowdsourcing implica una revolución de paradigma, aún poco entendida, dado que desestabiliza el principio mismo de robustez de la información, el cual fundamenta la legitimidad de las instituciones de gobierno generadoras de información oficial. En el transcurso del año 2014, varias juntas tuvieron lugar sobre este tema entre la ONG, el INEGI y otras instituciones gubernamentales, con el objetivo de formar mesas de trabajo permanentes sobre datos e indicadores. El objetivo de las mesas de trabajo intersectoriales encabezadas por la Secretaría de Desarrollo Agrario Territorial y Urbano (SEDATU) $y$ el INEGI, era de reunir en un mismo ejercicio a diferentes dependencias dedicadas a algún sector con impacto en el desarrollo del territorio, con expertos académicos y profesionales, para generar de manera integral y participativa metodologías de producción y explotación de los datos oficiales. Debido a un contexto institucional inestable, no se ha tenido información sobre la continuidad de estas mesas de trabajo intersectoriales. Sin bases institucionales lo suficientemente fuertes, el grupo de trabajo de la ONG no pudo dar continuidad esta gestión con el INEGI. Tampoco 
hemos tenido conocimiento de alguna forma de seguimiento por parte del INEGI sobre actividades de investigación con colaboración de instituciones, dependencias y sociedad civil.

Ante la falta de recursos de la ONG para seguir impulsando esta gestión, el grupo de trabajo consideró conveniente desarrollar de manera paralela un trabajo de largo plazo, con posibilidad de resultados más rápidos para avanzar en la generación de información. Por un lado, la información está en las manos de los usuarios de la ciudad, también usuarios de la tecnología en un número creciente. Por otro, lograr captar esta información representa un reto metodológico más que financiero. Iniciamos con estas observaciones nuestro recorrido en la información voluntaria o crowdsourcing. Dar paso a esta dinámica, sin experiencia ni método previo, sin herramienta, ni mecanismos comunitarios preexistentes para implementarlo, significaba experimentar desde cero y explorar en muchas direcciones. El objetivo era tener en un tiempo relativamente corto, resultados en cantidad suficiente para dar visibilidad a una problemática real, una visibilidad suficiente para generar un debate a nivel de la sociedad civil y de organizaciones de gobierno, en particular entre los actores generadoras de información oficial.

\section{Desarrollo de la plataforma Repubikla}

En este punto de la iniciativa, se encontraron intereses particulares y generales. Por un lado se realizó un análisis de las plataformas abiertas de mapeo existentes, estables, configurables, que nos permitieran construir una base de datos desde la comunidad, con un grado de complejidad que aporte un valor agregado y siempre accesible en línea con licencia abierta. Las plataformas de mapeo resultan ser muchas, pero ninguna convenía a nuestros criterios: una ergonomía atractiva, la mezcla de varios tipos de objeto (la mayoría permiten mapear una información de tipo puntual únicamente) y de datos complejos, de gestión personalizada de bases de datos, ni de potencial crecimiento del proyecto. Decidimos entonces iniciar un desarrollo tecnológico propio, aunque básico, que permitiera captar la información sobre las prácticas de movilidad de las personas de modo voluntario y gratuito, que las organizaciones profesionales del desarrollo urbano y las instituciones oficiales de estadísticas pudieran procesar con un costo casi nulo.

Debíamos construir la estrategia de captación de información. Un proyecto basado en la aportación voluntaria de la comunidad depende del acceso a una comunidad, y del convencimiento e interés de dicha comunidad para aportar información. Dentro del campo de la movilidad urbana, el que habíamos predefinido, debíamos entonces enfocar nuestro esfuerzo en un primer tiempo en una comunidad ya estructurada, sólida, y federada por intereses muy motivadores. El ciclismo, como subtema de la movilidad, apareció idóneo para orientar el esfuerzo de Repubikla, por el activismo con el cual cuenta en México, y por ser un subtema que 
sufre más que cualquier otro tema, de escasez de información y de falta de acción política. Se decidió aplicar la experiencia a este contexto particular, buscando con la comunidad existente una socialización más rápida, una mejor asimilación de la iniciativa, y una mejor adhesión al proyecto. En este sentido, un círculo con una fuerte movilización social representaba un contexto favorable, pues la plataforma además de servir a su objetivo particular de generación de datos para las políticas públicas, podía ser una herramienta útil para apoyar causas de comunicación y construcción de diálogo sobre políticas locales para los grupos, colectivos y asociaciones activistas.

Este primer interés temático encontró favorablemente un interés personal: ambos miembros del grupo de Repubikla son ciclistas y tenían una experiencia personal de las problemáticas de la movilidad en bicicleta en la ciudad, además de tener algunos contactos con la comunidad ciclista organizada.

Esta orientación temática puede ser vista como la mejor ocasión para consolidar la iniciativa de crowdsourcing cartográfico. En etapas futuras, la misma herramienta de mapeo o como ecosistema de plataformas, podría extenderse a más temas y grupos de usuarios: a los demás modos de desplazamiento no motorizados, a los modos motorizados, y más temas relativos o no a la movilidad urbana.

Se consideró en un inicio un desarrollo web independiente, progresando en una fase posterior hacia un desarrollo móvil. La primera fase es una plataforma web de mapeo disponible en cualquier navegador, ${ }^{1}$ para mapear itinerarios y puntos referidos a la infraestructura o algún incidente, así como zonas de la ciudad señaladas por alguna característica relacionada con la capacidad de transitar. A cada objeto creado en el mapa, se vincula un formulario de información estandarizada que puede acompañarse de variables personalizadas. La información completa alimenta una base de datos centralizada, en un servidor propio, descargable libremente bajo licencia del Instituto Tecnológico de Massachusetts (MIT por sus siglas en inglés). ${ }^{2}$ Dada la necesidad de enseñar directamente un resultado atractivo a un público no especializado en análisis de datos, la base de datos fue vinculada a un servicio de mapeo temático integrado a redes sociales. CartoDB, hoy Carto, fue la plataforma elegida para realizar un gran número de mapas atractivos visualmente, fácilmente personalizables y con zoom fijo sobre ciudades de interés. ${ }^{3}$

Este material queda disponible para las ciudades que quieran hacer uso del material directamente con fines de promoción de mejores condiciones urbanas para la movilidad ciclista o peatonal. El concepto completo de Repubikla contempla para un futuro cercano la construcción de un web service interconectado entre la aplica-

\footnotetext{
$<$ Repubikla.org $>$.

$<$ https://opensource.org/licenses/MIT>.

$<$ https://repubikla.carto.com/maps $>$.
} 
ción cartográfica y base de datos central, dispositivos web y móviles nuestros y de terceros, permitiendo la circulación y construcción de la información entre un ecosistema de aplicaciones existentes y por crearse.

Lograr obtener datos sobre la movilidad ciclista desde aplicaciones móviles de terceros además de una aplicación propia, permitiría obtener recorridos automáticamente georreferenciados, así como un gran número de informaciones sin necesidad de captura directa por parte del usuario: trazos de recorridos, tiempo, velocidad, información personal genérica. Vinculado a esta información automática, el punto de intercambio con las aplicaciones de terceros se ubica en la solicitud al usuario para capturar un complemento cualitativo de información: calificaciones o evaluaciones del recorrido y de la infraestructura. Las aplicaciones de interés entran en la categoría de navegación y planeación de viaje para el ciclista, ayudando al usuario a organizar su recorrido de un punto A a un punto B por el camino más adecuado. Algunas también ofrecen algunas métricas útiles para el usuario como, la georreferenciación de su ruta, su velocidad, el kilometraje acumulado, la pendiente y otros datos de rendimiento físico. Recientemente algunas otras aplicaciones han innovado, ofreciendo datos más útiles para el estudio de la movilidad, solicitando al usuario el motivo de su recorrido. Sin embargo, ninguna aplicación que sea a la vez conocida y utilizada por una amplia población de usuarios, ofrece datos descargables con una licencia abierta para uso de investigación, como lo hace Repubikla.

Considerando la economía de esfuerzo del usuario como clave para generar la información voluntaria, es necesario ampliar lo más posible el panorama de las fuentes de información, y no sólo limitarlo a nuestro desarrollo y herramienta. A su vez, contemplar la interacción de nuestras herramientas con un mundo de aplicaciones, así como el trueque de información con aplicaciones en su mayoría comerciales, implica tener una buena estrategia y definir un enfoque, tanto original como complementario, de otros intereses. Si este tema sigue en definición hoy día, parece evidente que la fuerza de Repubikla es su inserción proactiva en una comunidad de usuarios sensibilizada con el propósito de influir positivamente en la planeación urbana.

\section{Una definición comunitaria}

El trabajo de inserción en comunidades de usuarios apareció esencial desde un inicio. La aplicación no podía llegar a ningún resultado sin construir una comunidad de usuarios, lo cual se organizó como premisa del proyecto, a partir de la invitación al Congreso Anual de la Red de Colectivos Ciclistas Mexicanos, ${ }^{4}$ que tiene lugar en una ciudad distinta del país cada mes de noviembre. En una charla con un líder de 
esta red, una presentación de la herramienta ante la comunidad presente apareció oportuna. Se presentó la versión alpha de la aplicación web, planteando como un gran reto, la visibilización de las problemáticas ciclistas ante los tomadores de decisiones, con datos duros ciudadanos y mapas. En esta primera presentación se realizó también un mini taller de mapeo, que tenía la intención de recabar inicialmente cierta cantidad de datos.

Esta primera experiencia en el congreso ciclista resultó en un intercambio donde aprendimos más de nuestro público, que él de nosotros. El aprendizaje fue de tres niveles:

- Por un lado, nos permitió sondear el lenguaje, la recepción del sector activista ciclista mexicano a un tema científico sobre los datos y mapas; su percepción del rol potencial de este material en la toma de decisiones. Sondeamos su involucramiento en actividades orientadas a la toma de decisiones, y sus formas de colaborar con dinámicas internas y externas de liderazgos que determinan las dinámicas del sector, así como las posibilidades para una herramienta como Repubikla de integrarse a las acciones desarrolladas. Se trataba en este sentido, de descubrir y comprender empíricamente nuestro público, objeto y principal colaborador. Hasta la fecha seguimos adecuando el discurso, conceptos e ideas a este actor cambiante según cada contexto.

- El segundo aprendizaje fue sobre la necesidad clara de permanecer a la escucha y poder adaptarnos, continuamente, a las necesidades de este sector. Uno de los primeros comentarios expresados en la asamblea fue la discriminación que habíamos asumido en nuestro desarrollo conceptual, de las condiciones de movilidad del peatón. El interés polarizado de la asamblea también nos expresaba que los temas que nos parecían más importantes -el conocimiento de los motivos de la movilidad ciclista - no iban a federar lo suficiente el interés de los usuarios. Un método basado en la generación voluntaria de datos, con vista a captar grandes volúmenes de información, no puede depender de un esfuerzo permanente de justificación del tema de interés. Esta estrategia podría llevarse a cabo con resultados relativos, pero no puede ser la prioridad de un proyecto que busca imponerse como la principal herramienta de recolección y centralización de datos ciudadanos en un tema dado. Esta reflexión nos llevó a decidir adaptarnos a la corriente del sector ciclista, sea la que sea, siempre y cuando corresponda con una tendencia estructural en las líneas de acción del conjunto de este sector, y cuando apunta a tener un impacto, directo o indirecto en las políticas públicas. De manera más amplia inclusive, asumimos la idea que la plataforma podía prestarse a prácticas de mapeo en temas muy a la periferia del tema general de Repubikla, con el objetivo de difundir la herramienta en la sociedad civil organizada y seguir ampliando siempre nuestras perspectivas y entendimiento de los 
cuestionamientos y necesidades de las comunidades. Así mismo, la base de datos puede irse alimentando de informaciones que eventualmente resulten enriquecedoras en cierto análisis, por aportar materiales y enfoques distintos. En el registro de construcción de redes, el mantener permeables las fronteras conceptuales del proyecto, permite también diversificar la comunidad de usuarios y eventualmente inspirar en esta comunidad nuevas conexiones y alianzas que impacten sus propias iniciativas. Repubikla podría ser entendida entonces no solo como un receptor centralizado de información ciudadana, pero como observatorio y catalizador de acciones civiles.

Para responder a esta filosofía y enfoque conceptual, la base de datos de Repubikla y la interface de la herramienta de mapeo, fueron desarrolladas para permitir una gran flexibilidad en el tipo de informaciones y en los temas de trabajo. Cada objeto permite efectivamente la creación de campos personalizados que se traducen en conjunto en un sólo campo de texto, que se segmenta solamente al momento de la exportación de datos, mediante un script. Esta característica permitía entonces un uso personalizado y contextualizado de la herramienta: conservando un tronco común de información, cualquier individuo podía generar de manera adicional o sustituta, su propia estructura de información, aportando variables nuevas no contempladas por la herramienta. De esta manera, permitíamos la aportación de datos con valor diferencial según cada actor, incluido nuestro propio equipo, y de acuerdo con cada análisis. Al no imponer a nuestros usuarios una definición rígida de los temas de interés, podíamos pretender captar datos que nos parecen de alta relevancia sin que lo sean para los usuarios, sirviendo así a varios propósitos a la vez: se puede realizar un mapeo enfocado a sus temas más prioritarios, al mismo tiempo que se aporta complemento de información, el cual constituye para nuestro grupo el principal interés.

La cantidad de datos solicitados para un objeto dibujado, mediante formulario de preguntas, es siempre una cuestión crítica. Porque puede comprometer la participación voluntaria, es necesario limitar al máximo la extensión de la información requerida y el tiempo dedicado por el usuario a la herramienta. Esta característica vuelve muy compleja la evaluación de la relevancia de los datos solicitados "por default". La colaboración con un público muy diverso, que queremos numeroso, obliga también a razonar en términos de público promedio, donde las decisiones conceptuales tomadas no pueden apegarse a un solo perfil de nuestro público, pero tiene que ser razonablemente adecuada para la gran mayoría, basándonos en las características, tendencias e intereses más representados en estas comunidades.

- El tercer aspecto que se perfiló, desde la primera presentación y taller de mapeo con Repubikla, fue el obstáculo tecnológico, y la muy baja tolerancia del público a una tecnología en etapa de desarrollo, con las imperfecciones y debilidades 
de diseño que eso implica. La dificultad de atraer usos a la plataforma, las dificultades para entender el proceso a seguir, nos dio conciencia de los inconvenientes por enfrentar si no se contaba con un recurso financiero para acelerar su desarrollo. Los obstáculos principales serían una lenta adopción de la herramienta por los usuarios, lenta construcción de la base de datos, como resultado una baja credibilidad, y posiblemente una rápida pérdida de interés que podía ser fatal al proyecto.

Estos tres aspectos mencionados nos llevaron a mantener una presencia continua en el sector activista, en desarrollar temas vinculados de manera central y periférica al de los datos y del mapeo, tanto por nuestros propios intereses y por los de las personas y grupos con quienes participamos, tomando como oportunidad para socializar la herramienta pese a las debilidades y defectos temporales de ergonomía y diseño. Nos parecía siempre más claro, que entre más rápido producíamos y centralizábamos datos, más interés ganábamos, entre públicos más diversos, lo que podría ayudar en reducir impacto negativo de las limitaciones funcionales de la herramienta, la cual se seguía desarrollando a un ritmo más lento.

A su vez, la misma necesidad de presencia y aportación continua, así como las oportunidades de colaboración que han ido creciendo, no permitió dedicar el tiempo requerido al desarrollo de propuestas y candidaturas para el fondeo del desarrollo. Esta situación nos llevó a seguir una tendencia basada siempre en la comunidad y la colaboración, que en privilegiar el desarrollo tecnológico rápido, lo cual poco a poco cobraba sentido y coherencia con la filosofía misma del proyecto.

Dos años después, con un desarrollo en tres etapas - una nueva versión ha sido liberada en este mes de septiembre 2016-, con colaboraciones crecientes con la red de ciclismo urbano y con colectivos y organizaciones de ciudades específicas, aparece con una gran claridad la necesidad de adaptabilidad y de interconexión de la herramienta con su entorno tecnológico y social. Un desarrollo tecnológico temprano hubiera opacado los matices en los cuales debía fundarse la iniciativa para lograr su inserción comunitaria. A su vez, en lugar de haber dirigido nuestra atención y esfuerzo en el financiamiento del desarrollo, nos enfocamos en una mejor definición y al progreso de dinámicas colaborativas centrada en los datos - su producción, análisis y usos- junto con la comunidad. De esta manera, el proyecto se ha orientado hacia un experimento de gobernanza sobre datos de movilidad, indagando a profundidad los mecanismos de participación y de inserción de la información en procesos complejos de interacción entre actores de la sociedad civil e instituciones públicas. Esta orientación también conllevó al crecimiento de un grupo de trabajo representado por personas de perfiles distintos, vinculados al urbanismo y al análisis de datos, en acciones puntuales o generales que empieza a conformar, aunque de manera intermitente, un círculo espontáneo de aprendizaje. 